\title{
DERIVATIONS IN CENTRAL SEPARABLE ALGEBRAS
}

\author{
by GEORGE T. GEORGANTAS
}

(Received 24 August, 1976)

Introduction. Given $N$ a finite separable normal extension of a field $F$, it is well known that the Brauer group $\operatorname{Br}(N / F)$ of classes of central simple $F$-algebras split by $N$ is isomorphic with $\operatorname{Ext}\left(N^{*}, G\right)$, the classes of group extensions of $N^{*}$ by the Galois group $G$ of $N$ over $F$. In the construction of this isomorphism, a key role is played by the Skolem-Noether Theorem which extends automorphisms to inner automorphisms in central simple algebras.

An analogue holds for inseparable extensions $K$ over $F$. Jacobson [7, pp. 667-670] showed it was possible to have a crossed-product theory with derivations playing the role of automorphisms. He obtained a set-theoretic map between $F / Z$ and $\operatorname{Br}(K / F)$ by showing that derivations in central simple algebras extend to inner ones. Hochschild later showed there is actually an isomorphism between these two groups by also utilizing this extension of derivations. ([6, pp. 477-489]).

The notion of the Brauer group has been extended since then and is now defined for rings. The fact that derivations extend to inner derivations on central separable algebras is the key to obtaining the fact that, for $C$ a ring extension over $A$, the Brauer group $\operatorname{Br}^{\prime}(C / A)$ is isomorphic to a certain factor group of $A$, thus giving an additive description of $\mathrm{Br}^{\prime}(C / A)$, (see [5]).

In [11, pp. 427-450], Yuan showed that for $R$ a central separable $A$-algebra split by $C$, every higher $A$-derivation of $C$ into $R$ extends to an inner higher derivation of $R$. The fact that the analogous property is not true for automorphisms on central separable algebras (see [10, p. 1117]), i.e., that such automorphisms need not be inner, shows that we have a special situation meriting further study. In the case where the projective class group $\mathrm{P}(C / A)$ is trivial, Yuan $[11$, p. 430] has shown that every automorphism on a central separable $A$-algebra split by $C$ leaving $C$ pointwise fixed must be inner.

Background. An $A$-algebra $S$ is a ring $S$ together with a ring monomorphism of $A$ into the center of $S$. We call $S$ a central $A$-algebra if the image of this homomorphism is the center of $S$. The opposite $A$-algebra of $S$ is denoted by $S^{\circ}$. The enveloping algebra of $S$ over $A$, denoted by $S^{e}$, is the $A$-algebra $S \otimes_{A} S^{o}$.

Given an $S$-bimodule $M$, we may regard $M$ as a left $S^{e}$-module by defining $\left(s \otimes t^{\circ}\right) x=s x t$, where $x \in M ; s, t \in S$. Likewise, any left $S^{e}$-module can be regarded as an $S$-bimodule. In particular, $S$ has a left $S^{e}$-module structure. We call $S$ a separable $A$-algebra if $S$ is a projective $S^{e}$-module. Thus, a central separable $A$-algebra is a separable $A$-algebra whose center is $A$. It is well-known that $S$ is a central separable $A$-algebra if and only if $S^{e}$ is $\left(A\right.$-algebra) isomorphic to $\operatorname{End}_{\mathrm{A}}(S)$, and $S$ is finitely generated and projective as an $A$-module (see $[1$, p. 372]).

The notion of central separable algebras over commutative rings is a generalization of

Glasgow Math. J. 19 (1978) 75-77 
central simple algebras over fields. For, $S$ is a central separable $A$-algebra if and only if, for each maximal ideal $\eta$ of $A, S / \eta S$ is a central simple $A / \eta$-algebra.

Given $S$ a finitely generated projective $A$-module, let $\Omega=\operatorname{End}_{A}(S)$. If $M$ is any $\Omega$-module, the Morita Duality Theorem gives us an $\Omega$-isomorphism $\operatorname{Hom}_{\Omega}(S, M) \otimes_{A} S \cong$ $M$, (see [2, pp. 22-23]).

Let $B$ be a ring whose center is $A$, and let $S$ be an $A$-subalgebra of $B$. By considering $B$ canonically as a two-sided $S$-module, we can form $\{b \in B \mid b s=s b$ for all $s \in S\}$. We call this the commutant of $S$ in $B$ and denote it by $B^{S}$.

Main Result. Let $C$ be a commutative purely inseparable exponent one ring extension of $A$ with respect to a derivation $\partial$ on $C$, and let $C$ be finitely generated and projective as an $A$-module. In the following, $\Omega=\operatorname{End}_{C}(S)$ and $M^{C}$ denotes the commutant of $C$ in $M$. We shall make use of the Morita Duality Theorem to arrive at the result that derivations in central separable algebras are actually obtained from inner derivations on the algebras. This is stated in the following theorem.

We begin by taking $S$ to be any central separable $A$-algebra containing $C$ as a splitting $A$-subalgebra. We let $M=S \oplus S$ and we define a left $C \otimes_{\mathrm{A}} \boldsymbol{S}^{\circ}$-module structure on $M$ by $c\left(s_{1}, s_{2}\right)=\left(c s_{1}, \partial c \cdot s_{1}+c s_{2}\right)$ and $\left(s_{1}, s_{2}\right) t=\left(s_{1} t, s_{2} t\right)$, where $c \in C$ and $s_{1}, s_{2}, t \in S$.

Lemma 1. As right $C$-modules, $\operatorname{Hom}_{\Omega}(S, M) \cong M^{C}$.

Proof. The map $\mu: \operatorname{Hom}_{\Omega}(S, M) \rightarrow M^{C}$ defined by $\mu(f)=f(1)$ is a well-defined $C$ homomorphism since $\mu(1)$ commutes with each element of $C$. The verifications for $\mu$ being bijective are routine.

Lemma 2. Let $I=\left\{a \in S \mid \exists t \in S\right.$ such that $\left.(a, t) \in M^{C}\right\}$. Then $I$ is a non-zero ideal of $C$.

Proof. To see that $I$ is a subset of $C$, take any $a \in I$. Then there exists $b \in S$ such that $(a, b) c=c(a, b)$, for all $c \in C$. By equating the first components, we see that $a \in C$. It is straightforward to verify the remaining properties.

THEOREM . Given $C$ a commutative purely inseparable exponent one ring extension of $A$ with respect to a derivation $\partial$ on $C$, and given $C$ finitely generated and projective as an $A$-module, let $S$ be any central separable A-algebra containing $C$ as a splitting $A$-subalgebra. Then $\partial$ extends to an inner derivation on $S$.

Proof. Take the above $C \otimes_{A} S^{o}$-module structure on $M$, and consider $M$ canonically as an $\Omega$-module. Since $S$ is finitely generated and projective as a $C$-module, the Morita Duality Theorem [4, p. 37] gives us an $\Omega$-isomorphism $M \cong \operatorname{Hom}_{\Omega}(S, M) \otimes_{C} S$. By Lemma 1 , we thus obtain $M \cong M^{C} \otimes_{C} S$, with the isomorphism given by $m \otimes s \rightarrow m s$. Therefore, $S \oplus S=M^{C} \cdot S$.

Since $S$ is a finitely generated projective $C$-module containing $C$, for each maximal ideal $\beta$ of $C, S_{\beta}$ is a finite dimensional free $C_{\beta}$-module. Thus, we can form the canonical projections $\pi_{k}: S_{\beta} \rightarrow C_{\beta}$, and the canonical injections $i_{k}: C_{\beta} \rightarrow S_{\beta}$ split for each $k$. Then $S_{\beta}$ is faithfully flat over $C_{\beta}[9$, p. 333]. But Lemma 2 implies $I \subseteq I \cdot S \cap C$, so $I=I \cdot S \cap C$. 
Therefore, $I=I \cdot S \cap C=S \cap C=C$, so $1 \in I$. This means there exists $t \in S$ such that $c(1, t)=(1, t) c$, for all $c \in C$; i.e., we now have an element $t \in S$ such that $\partial c=t c-c t$, for all $c \in C$. Hence, $\partial$ is extended to an inner derivation on $S$.

\section{REFERENCES}

1. M. Auslander and $O$. Goldman, The Brauer group of a commutative ring, Trans. Amer. Math. Soc. 97 (1960), 367-409.

2. M. Auslander and O. Goldman, Maximal orders, Trans. Amer. Math. Soc. 97 (1960), 1-24.

3. N. Bourbaki, Algebre commutative, Chapts. 1, 2, Actualites Sci. Indust. 1290 (Hermann, Paris, 1961).

4. S. Chase and A. Rosenberg, Amitsur cohomology and the Brauer group, Mem. Amer. Math. Soc. 52 (1965), 34-79.

5. G. Georgantas, Inseparable Galois cohomology, J. Algebra, 38 (1976), 368-379.

6. G. Hochschild, Simple algebras with purely inseparable splitting fields of exponent 1, Trans. Amer. Math. Soc. 79 (1955), 477-489.

7. N. Jacobson, p-algebras of exponent p, Bull. Amer. Math. Soc. 43 (1937), 667-670.

8. A. Rosenberg and D. Zelinsky, Amitsur's complex for inseparable fields, Osaka Math. J. 14 (1962), 219-240. $327-356$.

9. A. Rosenberg and D. Zelinsky, On Amitsur's complex, Trans. Amer. Math. Soc. 97 (1960),

10. A. Rosenberg and D. Zelinsky, Automorphisms of separable algebras, Pacific J. Math. 11 (1961), 1109-1117.

11. S. Yuan, Central separable algebras with purely inseparable splitting rings of exponent one, Trans. Amer. Math. Soc. 153 (1971), 427-450.

Department of Mathematics

ROCHESTER INSTITUTE OF TECHNOLOGY

1 Lomb Memorial Drive

ROCHESTER, NEW YORK 14623 